\title{
Secular trends in cesarean sections and risk factors in South Korea (2006-2015)
}

\author{
Ho Yeon Kim, PhD', Dokyum Lee, MD¹, Jinsil Kim, MD², Eunjin Noh, Bachelor ${ }^{2}$, Ki-Hoon Ahn, PhD,
} Soon-Cheol Hong, PhD', Hai-Joong Kim, PhD', Min-Jeong Oh, PhD', Geum Joon Cho, MD, PhD

${ }^{1}$ Department of Obstetrics and Gynecology, Korea University College of Medicine, ${ }^{2}$ Korea University Guro Hospital Smart Healthcare Center, Seoul, Korea

\section{Objective}

This study aimed to investigate trends in the rate of cesarean sections (CSs) in South Korea from 2006 to 2015 and identify the risk factors associated with these changes.

Methods

Using the National Health Insurance Corporation dataset, all women who gave birth between 2006 and 2015 were included in the study. We investigated 1) the mode of delivery, 2) the complication rates during pregnancy (i.e., preeclampsia and placenta previa), and 3) pre-pregnancy factors (body mass index, hypertension [HTN], diabetes mellitus [DM], and other pre-existing medical conditions), and their trends during the study period.

Results

Over 10 years, the rate of CS increased from 36.3\% in 2006 to $40.6 \%$ in $2015(P<0.01)$. The rate of CS increased in primiparous women, women with multiple pregnancy, and women with preeclampsia. Maternal age and the incidence of placenta previa also increased. In contrast, the rate of vacuum deliveries and vaginal birth after CS decreased during the study period. The rate of women with pre-pregnancy obesity and DM increased, but the rate of women with pre-pregnancy HTN decreased.

\section{Conclusion}

The rate of CS in South Korea increased from 2006 to 2015. This trend may reflect changes in the rate of different risk factors. Identifying the causes of the increasing CS trend observed in this study will allow clinicians to monitor these factors and possibly reduce the rate of CS.

Keywords: Cesarean section; Trends; Risk factors

\section{Introduction}

Cesarean sections (CSs) are the most common major surgical procedure for women. There is no doubt that CS is a lifesaving procedure for mothers and babies. This procedure can prevent adverse pregnancy outcomes such as perinatal asphyxia, stillbirth, uterine rupture, or obstetric fistula [1]. However, an increase in the use of CS is worrisome in many ways. The World Health Organization (WHO) reported that the rate of CS is increasing and nearly doubled between $2000(12.1 \%)$ and 2015 (21.1\%) [2,3]. CSs are associated with not only higher economic burden than those by vaginal births but also with health burden such as the risk of uterine rupture, abnormal placentation, ectopic pregnancy, stillbirth,
Received: 2019.11.13. Revised: 2020.02.28. Accepted: 2020.03.02. Corresponding author: Geum Joon Cho, $\mathrm{MD}, \mathrm{PhD}$

Department of Obstetrics and Gynecology, Korea University College of Medicine, 148 Gurodong-ro, Guro-gu, Seoul 08308, Korea

E-mail: geumjoon@korea.ac.kr

https://orcid.org/0000-0001-6761-0944

Articles published in Obstet Gynecol Sci are open-access, distributed under the terms of the Creative Commons Attribution Non-Commercial License (http://creativecommons. org/licenses/by-nc/3.0/) which permits unrestricted non-commercial use, distribution, and reproduction in any medium, provided the original work is properly cited.

Copyright $\odot 2020$ Korean Society of Obstetrics and Gynecology 


\section{Obstetrics \& Gynecology Science}

Ho Yeon Kim, et al. Cesarean section in South Korea

preterm birth, and altered immune systems and obesity in children born through a CS [4].

The CS rate in South Korea ranks fifth among 24 countries in the Organization for Economic Cooperation and Development [5]. The rate of CS in other East Asian countries is less by $10 \%$, although women in these countries share similar pelvic cavity characteristics. There is no definite consensus on the optimal rate of CS. However, the WHO recommends a rate of CS of approximately 10-15\% because the maternal and neonatal health risks outweigh the benefits beyond $15 \%[6]$.

Understanding the trends in the factors affecting cesarean deliveries may benefit from assessing the effects of both maternal and healthcare provider characteristics on the change in CS rates and assist in designing effective interventions to optimize the rate of CS. Therefore, we examined recent trends in CS and the related factors that influence its rate.

\section{Materials and methods}

\section{Study data}

Study data were collected by merging the Korea National Health Insurance (KNHI) claims database with the National Health Screening Examination (NHSE) data.

In South Korea, $97 \%$ of the population is required to enroll in the $\mathrm{KNHI}$ program. The remaining $3 \%$ of the population is covered by the medical aid program. Thus, using the KNHI claims dataset, which contains information on all claims, nearly all information about diseases and their treatment can be identified from this centralized database, with the exception of procedures not covered by insurance.

As part of the KNHI healthcare system, all insurance subscribers and dependents are invited to participate in a biannual NHSE free of charge. The NHSE consists of $2 \mathrm{com}-$ ponents: a health interview and a health examination. Therefore, pre-pregnancy information contained in the NHSE was also used in this study.

\section{Study population}

Based on the KNHI claims dataset, we identified all women who delivered between January 1, 2006 and December 31, 2015 (dataset 1). Using dataset 1, the delivery mode, pregnancy outcomes, and pre-pregnancy diseases were identified based on the International Classification of Diseases, 10th
Revision (ICD-10) codes.

Women were included in the analysis if they had an NHSE 1 to 2 years before their delivery (dataset 2). Pre-pregnancy characteristics were evaluated in dataset 2 .

\section{Measurements of outcomes and pre-pregnancy and pregnancy characteristics}

The mode of delivery was identified using electronic data interchange (EDI) codes in the $\mathrm{KNHI}$ claims database using dataset 1.

Pregnancy characteristics and pre-pregnancy diseases were evaluated using dataset 1. Information on pregnancy characteristics including primiparity, multiple pregnancy, instrumental delivery, vaginal birth after CS (VBAC), placenta previa, and preeclampsia was collected using EDI codes and ICD-10 codes. Information on pre-pregnancy diseases including diabetes mellitus (DM; E10-E14), hypertension (HTN; I10-I15), renal diseases (N10-N08, N10-N16), and cardiovascularpulmonary diseases (I20-128, 160-169), hypothyroidism (E02E03), hyperthyroidism (E05), and systemic diseases (M30M36) was retrieved based on the ICD-10 codes.

Information on pre-pregnancy characteristics including smoking status, obesity, fasting glucose, liver function tests (LFTs), and total cholesterol (TC) levels was collected using dataset 2. Smoking status was categorized into current smokers, past smokers, and never smokers. Health examinations included the measurement of body mass index (BMI) in kilograms per square meter. Obesity was defined as BMI $\geq 25$ $\mathrm{kg} / \mathrm{m}^{2}$ [7]. All blood samples were obtained after a minimum fasting period of 8 hours. The levels of fasting glucose, aspartate aminotransferase (AST), alanine aminotransferase (ALT), and cholesterol were measured using enzymatic methods. A high fasting glucose level was defined as glucose $\geq 126 \mathrm{mg} / \mathrm{L}$ [8]. High LFTs were defined as AST and ALT levels $>31 \mathrm{U} / \mathrm{L}$ [9]. High cholesterol was defined as cholesterol $\geq 200 \mathrm{mg} / \mathrm{L}$ [10].

\section{Statistical analyses}

The data are expressed as mean \pm standard deviation for continuous variables and as percentage for categorical variables.

Clinical and biochemical characteristics by delivery years were compared using analysis of variance (ANOVA) for differences in continuous variables and using the $\chi^{2}$ test for categorical variables. Secular trends in the continuous and categorical variables were determined and compared across the years using ANOVA polynomial regression tests and chi- 


\section{Obstetrics \& Gynecology Science}

Vol. 63, No. 4, 2020

square Cochran-Armitage tests, respectively. All tests were 2-sided, and $P<0.05$ was considered to indicate statistical significance. Statistical analyses were performed using SAS for Windows, version 9.4 (SAS Inc., Cary, NC, USA).

\section{Results}

During the study period, 4,190,949 deliveries were recorded.

\section{Trend in cesarean section from $\mathbf{2 0 0 6}$ to $\mathbf{2 0 1 5}$}

The rate of CS increased from $36.3 \%$ in 2006 to $40.6 \%$ in 2015, but unchanged from 2006 to 2011, and with a continuous increase each year after $2011(P<0.01)$ (Fig. 1).

\section{Trend in pregnancy factors related to cesarean section}

The secular changes in maternal characteristics and pregnancy factors related to CS are summarized in Table 1. The mean maternal age at delivery increased from 29.92 years in 2006 to 31.85 in 2015. Likewise, the proportion of mothers who had advanced age ( $\geq 35$ years and $\geq 40$ years) increased. There were no changes in the rate of underage ( $<20$ years) mothers during the study period. The rate of primiparity and multiple pregnancy increased, and the rate of CS increased in these high-risk groups. The number of vacuum deliveries and VBACs decreased during the study period. The prevalence of placenta previa increased during the study period.

\section{Trend in pre-pregnancy diseases}

Table 2 shows the secular changes in the prevalence of

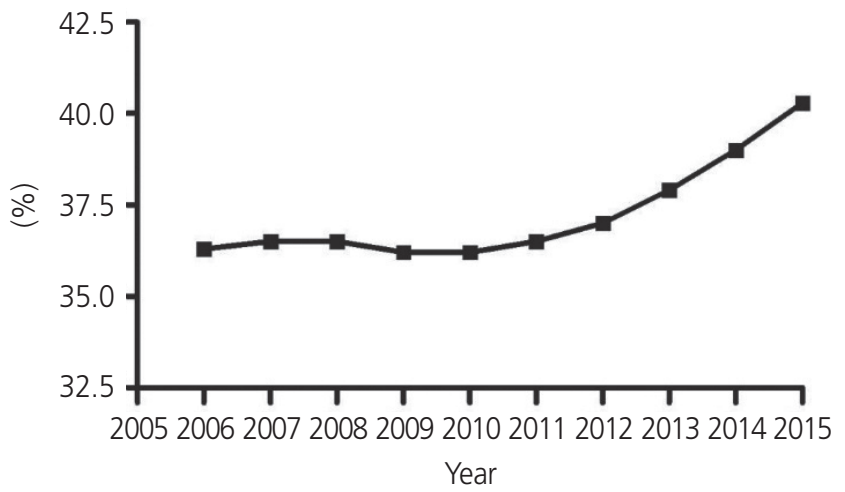

Fig. 1. Secular trends in the cesarean section rate between 2006 and 2015 .

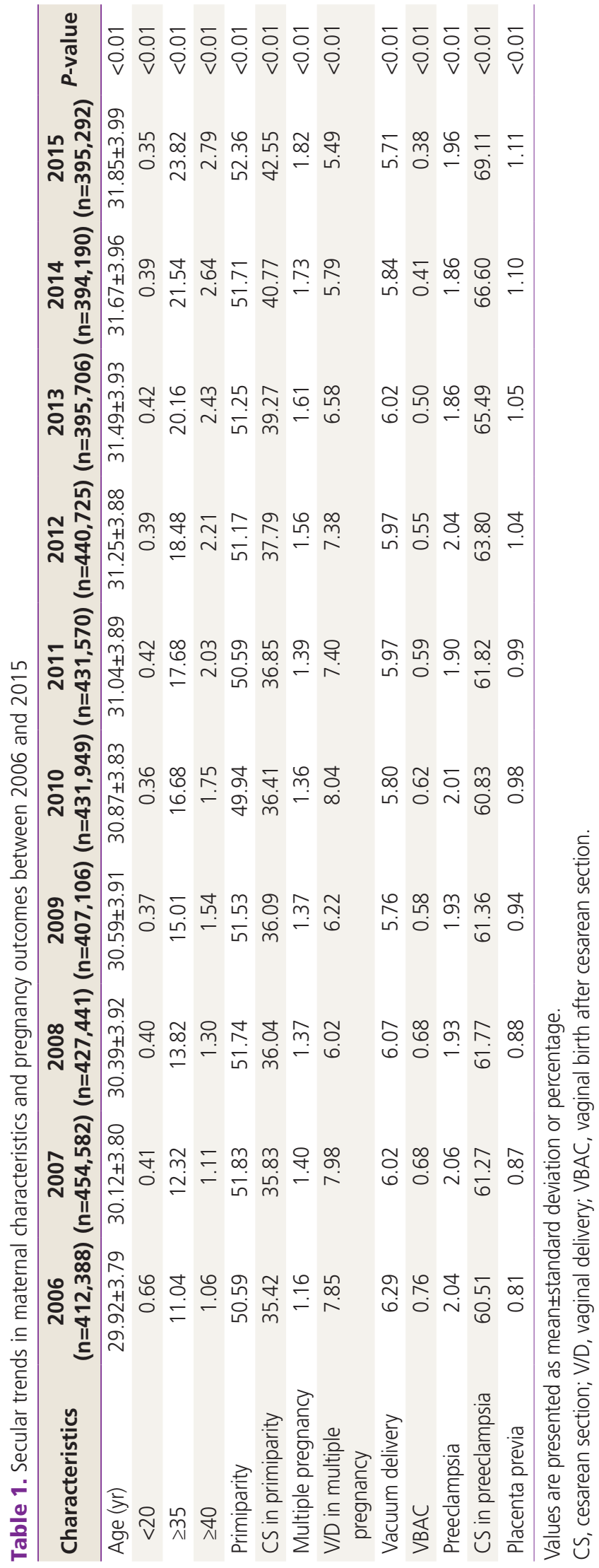




\section{Obstetrics \& Gynecology Science}

Ho Yeon Kim, et al. Cesarean section in South Korea

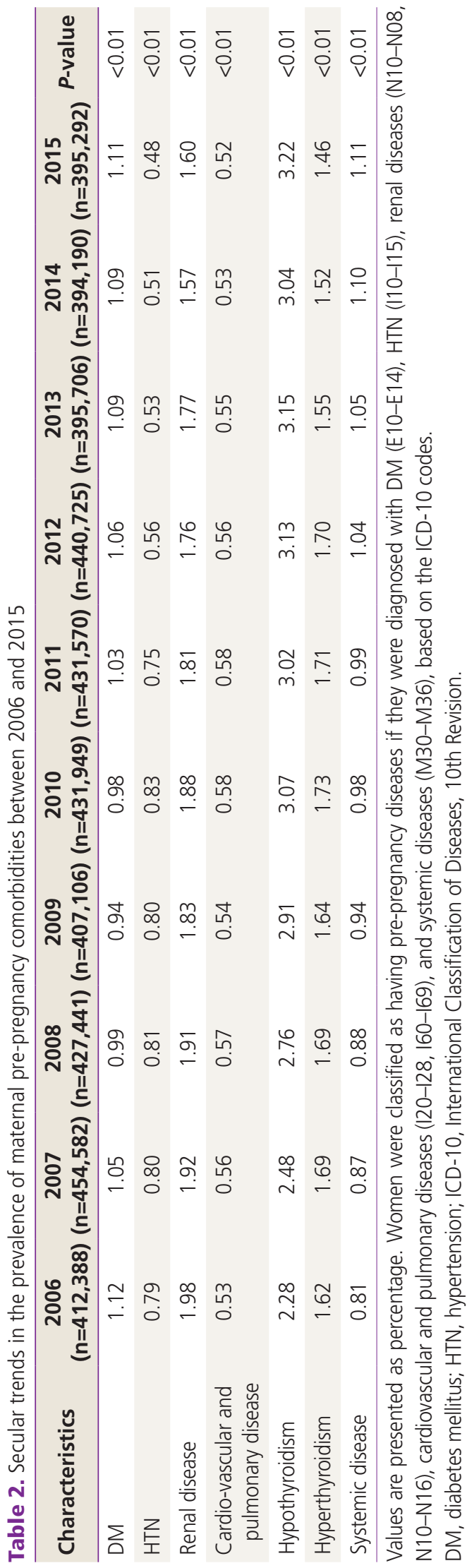

maternal pre-pregnancy diseases. The prevalence of prepregnancy DM, cardiovascular-pulmonary disease, hyperthyroidism, and systemic disease was unchanged, but that of HTN and renal disease decreased during the study period. The prevalence of hypothyroidism increased.

\section{Trend in pre-pregnancy factors related to cesarean section}

The secular changes in pre-pregnancy risk factors related to CS were identified, as summarized in Table 3. The prevalence of pre-pregnancy obesity increased from $7.13 \%$ in 2006 to $10.67 \%$ in 2015 . The prevalence of pre-pregnancy abnormal LFTs, high fasting glucose, and high TC also increased during the study period. The prevalence of pre-pregnancy smokers also increased.

\section{Discussion}

In this study, we evaluated secular trends in the rates of CS deliveries from 2006 to 2015 and their increase from 36.5\% in 2007 to $40.3 \%$ in 2015. A previous study on changes in CS rate South Korea from 1982 to 2012 reported that the rate remained at $36 \%$ for the most recent 6 years of the study [11]. Our study evaluated 3 later years than that study and found that the rate increased significantly up to $40 \%$. Increased maternal age, primiparity, pre-pregnancy obesity, multiple pregnancy, and placenta previa were contributing factors to the increase, while the rate of vacuum deliveries and VBACs decreased during the study period.

The rise in the rate of CS is a worldwide phenomenon. A multinational survey of the CS rates by the WHO reported an overall mean of $26.4 \%$ [12], and a recent global analysis of 150 countries reported a $12.4 \%$ increase in the rate of CS, with an average annual rate of increase of $4.4 \%$ over the past decades [13]. The trend in CS in Hong Kong, whose population shares similar pelvic sizes as that in our population, demonstrated a $10 \%$ increase during a 20 -year study period, resulting in CS rates up to 24-25\% [14]. European countries including Norway and England also showed moderate increases of $15.4 \%$ and $24.1 \%$, respectively [15]. Our study found a significantly increasing rate over the study period, almost 3 times higher than that in 2015 when compared to the WHO-recommended CS rate. Herein, we explored the factors contributing to the increase in the rates of CS. 


\section{Obstetrics \& Gynecology Science}

Vol. 63, No. 4, 2020

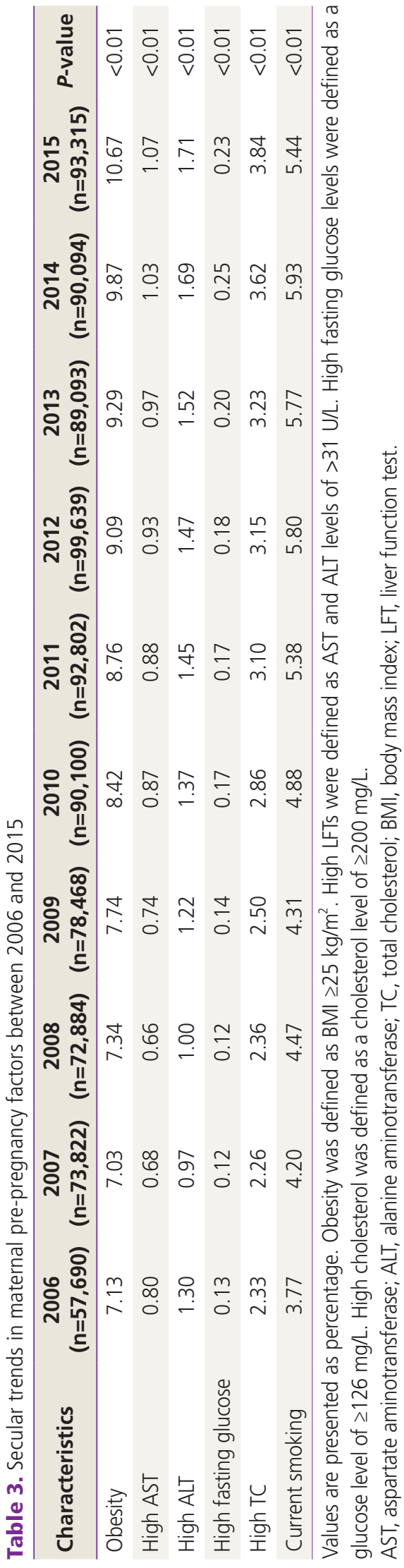

One important finding to consider is the increase in the rate of primary CS. This can lead to another CS in the future because VBACs have been decreasing as shown in our results. The risk of uterine rupture increases with VBAC attempts, and this catastrophic event may lead to litigation problems. In addition, the incidence of placenta previa was increased in our population. Placenta previa might be the cause for the rise in rates of CS, or the increased the rates of CS might be the cause of placenta previa. The risk of placenta previa in subsequent pregnancies increases with the number of previous cesarean deliveries $[16,17]$. Increased maternal age, in vitro fertilization, and previous myomectomy are also directly linked to the risk of placenta previa $[18,19]$.

One cause of increased primary CS is maternal request without indication. A previous survey conducted in Korea showed that $6-7 \%$ of CS were preformed upon maternal request without indication $[11,20]$. The increase in women's dissatisfaction with long labor and vaginal delivery and fear of emergency CS in the middle of labor due to arrest of labor or failure to progress have resulted in lower obstetrician thresholds for CS deliveries. The decrease in operative deliveries using forceps or vacuum was another consequence observed in our population. As operative delivery is associated with pelvic floor injuries and acute perinatal injuries, obstetricians tend to perform CS instead of using forceps or vacuum. The society has generally become far less tolerant of poor outcomes, and a trend has been toward placing blame either on the individual or on the system involved. This condition, and the fear of litigation, makes clinicians reluctant to resist maternal requests.

The most pressing social issue in South Korea is the low birth rate, which was only 0.98 in 2018 [21]. The main reasons contributing to this situation are women balancing careers with having children, the high cost of raising children, lifestyle choices regarding marriage, gender equity values, and higher education levels of women [22]; people avoid getting married and having many babies. This social phenomenon results in increased nulliparity and maternal aging. Consistent with previous findings, maternal aging, multiple pregnancy, and pre-pregnancy obesity were associated with increased rates of CS in this study [23].

There has been a gradual increase in the mean age of pregnant women in Korea. Older maternal age leads to a further rise in the CS rate. Maternal aging is a consequence of women's higher education level, followed by marriage at an older 


\section{Obstetrics \& Gynecology Science}

Ho Yeon Kim, et al. Cesarean section in South Korea

age. Increased maternal age poses higher obstetric complications because these women have more chronic diseases such as diabetes and HTN before and during pregnancy. The rate of preeclampsia was slightly decreased during 10 years, but a higher rate of CS in preeclamptic patients was observed. One possible explanation for the decrease in preeclampsia is the introduction of voucher programs for maternal health care covered by KNHI since 2008, and this program has allowed easier access to facility antenatal care and reduced the financial burden of low-income pregnant women [24]. Maternal aging also results in an increased failure to progress due to a contracted pelvis [25]. Women with contracted pelvis are likely to pass their pelvic dimensions to their own offspring. This phenomenon implies that the application of life-saving CS can evolutionarily increase the rates of contracted pelvises [26]. These factors related to aging lead to increased rates of CS. Several previous statistics in many countries showed the link between older maternal age and the increased rates of CS [27].

Our results showed a $50 \%$ increase in the frequency in multiple pregnancy and decreased numbers of vaginal deliveries in multiple pregnancy. The evolution of infertility therapy is one of the main reasons for the increase in multiple pregnancy. In Korea, the Ministry of Health and Welfare has established regulations on the number of embryo transfers regarding maternal age and status of embryos such that no more than 3 cleavage-stage embryos or 2 blastocysts can be transferred even after the age of 35 . This has had a direct influence on adverse obstetrical outcomes including increase in the rate of CS deliveries. There are more chances of malpresentation in multiple pregnancy and even without malpresentation, and doctors tend to perform CS instead of vaginal delivery.

Several pre-pregnancy factors were associated with increased rates of CS deliveries. Pre-pregnancy obesity was one important factor that increased the rate of CS. There has been a rapid rise in maternal pregnancy BMIs in the past decades, and the prevalence of adult obesity in South Korea increased from $29.7 \%$ in 2009 to $32.4 \%$ in 2015 due to increase in childhood obesity and human microbiome issues, in agreement with our results $[28,29]$. Obesity is a well-known factor that contributes to adverse maternal and neonatal outcomes [30]. Associated factors that lead to CS are chronic diseases of the mother, macrosomia due to uncontrolled blood glucose levels, excess intra-abdominal adipose tissue causing obstructed labor, and induction agent intolerance due to large body volumes [31-33].

Despite the decreasing trend in smoking among people worldwide, our results showed that smoking prevalence among Korean women increased [34]. Changes in gender role, higher education, and social norms are contributing factors for the increase in smoking trend among women. Other than this factor, abnormal liver function and higher fasting glucose levels appear to be associated with the risk of $\mathrm{CS}$. These factors may increase the rate of CS because they are strongly associated with comorbidities. However, the relationship between these factors and CS should be investigated in the future.

Several interventions have been implemented to reduce unnecessary CS, but their effectiveness was limited. However, counseling low-risk primipara mothers who favor CS on the risk of CS and educating them on optimal gestational weight gain and the importance of controlling body weight before pregnancy may help to prevent unnecessary CS. In low-risk pregnancies, women who want a primary CS should be counseled on the increased risks of perinatal mortality and morbidity resulting from CS. They include higher blood loss, infection rates, thromboembolism, and adjacent organ injuries in mothers and respiratory problems and mortality in babies $[35,36]$. One previous study conducted in China reported that attendance in a prenatal education course significantly reduced the rate of maternal CS requests [37]. Education on labor and normal delivery, the benefit of babies born vaginally, the perinatal risk of CS, pain management during labor, and the supporting roles of the husband or parents by skilled obstetricians, and maternal unit nurses or midwives may help to lower the number of CS as noted in this study.

This population-based cohort study reports trends in the rate of CS deliveries in South Korea over 10 recent years. However, the study had several limitations. First, social factors such as educational background, socioeconomic status, labor force participation, and publicity of the hospital, which are known to be associated with CS rates, were not considered. Second, the results of our study are not completely generalizable because our health insurance system is different from those in other countries and the population enrolled was East Asian. Third, the indications for primary CS were not described and may not be accurate because insurance does not cover the CS on maternal request.

We observed increasing trends in the rates of CS over the 


\section{Obstetrics \& Gynecology Science}

Vol. 63, No. 4, 2020

recent decade. Maternal aging, increased primiparity, increased pre-pregnancy obesity, increased placenta previa, increased multiple pregnancy, and decreased vacuum deliveries and VBACs were major factors associated with the increased rates of CS. CS should be undertaken when medically necessary, and informed consent should include short-term and long-term effects of CS for the sake of the mother and the baby. Efforts should focus on providing CS to the appropriate women at the right time. In addition, public health programs to lower the rate of CS should be established, and multidisciplinary discussion should be performed in the future, whether national health insurance will cover "CS upon maternal request."

\section{Conflict of interest}

No potential conflict of interest relevant to this article was reported.

\section{Ethical approval}

This study was approved by the Institutional Review Board (IRB) of the Korea University Medical Center (IRB No. 2019GR0256).

\section{Patient consent}

All the information that was provided for the study had been de-identified; therefore, we were unable to obtain informed consents from the participants.

\section{References}

1. Ahmed S, Tunçalp Ö. Burden of obstetric fistula: from measurement to action. Lancet Glob Health 2015;3:e243-4.

2. Boerma T, Ronsmans C, Melesse DY, Barros AJ, Barros $F C$, Juan $L$, et al. Global epidemiology of use of and disparities in caesarean sections. Lancet 2018;392:1341-8.

3. Betrán AP, Temmerman $M$, Kingdon C, Mohiddin A, Opiyo N, Torloni MR, et al. Interventions to reduce un- necessary caesarean sections in healthy women and babies. Lancet 2018;392:1358-68.

4. Sandall J, Tribe RM, Avery L, Mola G, Visser GH, Homer CS, et al. Short-term and long-term effects of caesarean section on the health of women and children. Lancet 2018;392:1349-57.

5. OECD. Health at a glance 2013: OECD indicators [Internet]. Paris: OECD Publishing; 2013. Available from: https://www.oecd.org/els/health-systems/Health-at-aGlance-2013.pdf.

6. Appropriate technology for birth. Lancet 1985;2:436-7.

7. Finucane MM, Stevens GA, Cowan MJ, Danaei G, Lin JK, Paciorek CJ, et al. National, regional, and global trends in body-mass index since 1980: systematic analysis of health examination surveys and epidemiological studies with 960 country-years and 9.1 million participants. Lancet 2011;377:557-67.

8. Danaei G, Finucane MM, Lu Y, Singh GM, Cowan MJ, Paciorek CJ, et al. National, regional, and global trends in fasting plasma glucose and diabetes prevalence since 1980: systematic analysis of health examination surveys and epidemiological studies with 370 country-years and 2.7 million participants. Lancet 2011;378:31-40.

9. Clark JM, Brancati FL, Diehl AM. The prevalence and etiology of elevated aminotransferase levels in the United States. Am J Gastroenterol 2003;98:960-7.

10. Farzadfar F, Finucane MM, Danaei G, Pelizzari PM, Cowan MJ, Paciorek CJ, et al. National, regional, and global trends in serum total cholesterol since 1980: systematic analysis of health examination surveys and epidemiological studies with 321 country-years and 3.0 million participants. Lancet 2011;377:578-86.

11. Chung SH, Seol HJ, Choi YS, Oh SY, Kim A, Bae CW. Changes in the cesarean section rate in Korea (19822012) and a review of the associated factors. J Korean Med Sci 2014;29:1341-52.

12. Vogel JP, Betrán AP, Vindevoghel N, Souza JP, Torloni MR, Zhang J, et al. Use of the Robson classification to assess caesarean section trends in 21 countries: a secondary analysis of two WHO multicountry surveys. Lancet Glob Health 2015;3:e260-70.

13. Betrán AP, Ye J, Moller AB, Zhang J, Gülmezoglu AM, Torloni MR. The increasing trend in caesarean section rates: global, regional and national estimates: 19902014. PLoS One 2016;11:e0148343. 


\section{Obstetrics \& Gynecology Science}

Ho Yeon Kim, et al. Cesarean section in South Korea

14. Chung WH, Kong CW, To WW. Secular trends in caesarean section rates over 20 years in a regional obstetric unit in Hong Kong. Hong Kong Med J 2017;23:340-8.

15. Tollånes MC, Thompson JM, Daltveit AK, Irgens LM. Cesarean section and maternal education; secular trends in Norway, 1967-2004. Acta Obstet Gynecol Scand 2007;86:840-8.

16. Thurn $L$, Lindqvist $P G$, Jakobsson $M$, Colmorn LB, Klungsoyr $\mathrm{K}$, Bjarnadóttir RI, et al. Abnormally invasive placenta-prevalence, risk factors and antenatal suspicion: results from a large population-based pregnancy cohort study in the Nordic countries. BJOG 2016;123:1348-55.

17. Marshall NE, Fu R, Guise JM. Impact of multiple cesarean deliveries on maternal morbidity: a systematic review. Am J Obstet Gynecol 2011;205:262.e1-8.

18. Jauniaux E, Grønbeck L, Bunce C, Langhoff-Roos J, Collins SL. Epidemiology of placenta previa accreta: a systematic review and meta-analysis. BMJ Open 2019;9:e031193.

19. Tanimura K, Morizane M, Deguchi M, Ebina Y, Tanaka $U$, Ueno $Y$, et al. A novel scoring system for predicting adherent placenta in women with placenta previa. Placenta 2018;64:27-33.

20. Kim HK. Impact factors of Korean women's cesarean section according to ecological approach. Korean J Women Health Nurs 2011;17:109-17.

21. Statistic Korea. Statistics of birth and death, 2018. Daejeon: Statistic Korea; 2019.

22. Song JE, Ahn JA, Lee SK, Roh EH. Factors related to low birth rate among married women in Korea. PLoS One 2018;13:e0194597.

23. Nkoka O, Ntenda PA, Senghore T, Bass P. Maternal overweight and obesity and the risk of caesarean birth in Malawi. Reprod Health 2019;16:40.

24. Choe SA, Min HS, Cho SI. Decreased risk of preeclampsia after the introduction of universal voucher scheme for antenatal care and birth services in the Republic of Korea. Matern Child Health J 2017;21:222-7.

25. Kim SY, Park JY, Bak SE, Jang YR, Wie JH, Ko HS, et al. Effect of maternal age on emergency cesarean section. J Matern Fetal Neonatal Med 2019:1-8.

26. Pavličev M, Romero R, Mitteroecker P. Evolution of the human pelvis and obstructed labor: new explanations of an old obstetrical dilemma. Am J Obstet Gynecol 2020;222:3-16.

27. Bayrampour H, Heaman M. Advanced maternal age and the risk of cesarean birth: a systematic review. Birth 2010;37:219-26.

28. Ng M, Fleming T, Robinson M, Thomson B, Graetz N, Margono $C$, et al. Global, regional, and national prevalence of overweight and obesity in children and adults during 1980-2013: a systematic analysis for the Global Burden of Disease Study 2013. Lancet 2014;384:76681.

29. Lee M. Research trends in obesity \& obesogenic environments in Korea. Nutr Res Pract 2019;13:461-72.

30. Ovesen P, Rasmussen S, Kesmodel U. Effect of prepregnancy maternal overweight and obesity on pregnancy outcome. Obstet Gynecol 2011;118:305-12.

31. Arrowsmith S, Wray S, Quenby S. Maternal obesity and labour complications following induction of labour in prolonged pregnancy. BJOG 2011;118:578-88.

32. Al-Kubaisy W, Al-Rubaey M, Al-Naggar RA, Karim B, Mohd Noor NA. Maternal obesity and its relation with the cesarean section: a hospital based cross sectional study in Iraq. BMC Pregnancy Childbirth 2014;14:235.

33. Gaudet L, Wen SW, Walker M. The combined effect of maternal obesity and fetal macrosomia on pregnancy outcomes. J Obstet Gynaecol Can 2014;36:776-84.

34. Chang Y, Kang HY, Lim D, Cho HJ, Khang YH. Longterm trends in smoking prevalence and its socioeconomic inequalities in Korea, 1992-2016. Int J Equity Health 2019;18:148.

35. Malloy MH. Impact of cesarean section on intermediate and late preterm births: United States, 2000-2003. Birth 2009;36:26-33.

36. Saeed KB, Greene RA, Corcoran P, O'Neill SM. Incidence of surgical site infection following caesarean section: a systematic review and meta-analysis protocol. BMJ Open 2017;7:e013037.

37. Gao Y, Tang Y, Tong M, Du Y, Chen Q. Does attendance of a prenatal education course reduce rates of caesarean section on maternal request? A questionnaire study in a tertiary women hospital in Shanghai, China. BMJ Open 2019;9:e029437. 